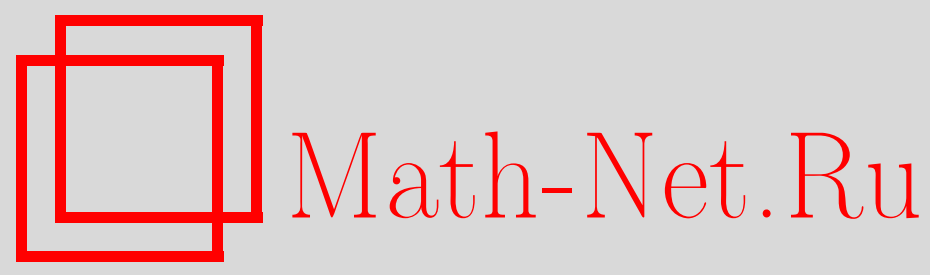

Е. Г. Скляренко, О локально свободных абелевых группах, Матем. заметки, 2005, том 77, выпуск 1, 121-126

DOI: https://doi.org/10.4213/mzm2475

Использование Общероссийского математического портала Math-Net.Ru подразумевает, что вы прочитали и согласны с пользовательским соглашением http://www. mathnet.ru/rus/agreement

Параметры загрузки:

IP: 18.207 .199 .55

26 апреля 2023 г., 17:58:08

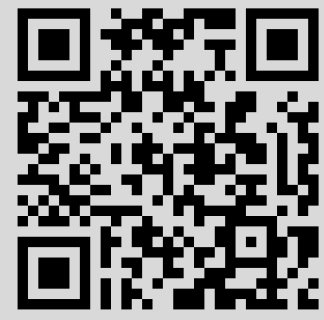




\section{О ЛОКАЛЬНО СВОБОДНЫХ АБЕЛЕВЫХ ГРУППАХ}

\section{Е.Г. Скляренко}

В статье доказан следующий результат: абелева группа $A L w_{1}, w$-эквивалентна свободной абелевой группе счетного ранга тогда и только тогда, когда она является счетно свободной абелевой группой.

Библиограффия: 14 названий.

Группа, в которой все конечно порожденные подгрупшы свободны, называется локально свободной $[1, \S 37 \mathrm{a}]$. Предназначенное для некоммутативных групп, это определение, очевидно, не представляет интереса в классе абелевых групп. В настоящей работе рассматриваются исключительно абелевы групы, не имеющие кручения. Такую группу $\Sigma$ будем называть локально свободной, если в ней свободны все подгрушшы конечного ранга. Эквивалентно, для любой конечно порожденной подгрушпы $E \subset \Sigma$ все содержащие $E$ подгруппы $M \subset \Sigma$, имеющие тот же ранг, что и $E$, свободны.

В этом определении нельзя ограничиться подгруппами ранга 1: существуют группы без кручения ранга 2, не являюшиеся свободными, все подгрупшы ранга 1 в которых свободные циклические (ср. с теоремой 88.4 в [2]).

В настоящей работе доказывается, что локально свободные абелевы групшы - это в точности пределы простых индуктивных систем абелевых групп. Индуктивная система групп $\left\{M_{\lambda}, i_{\mu}^{\lambda}\right\}$ называется простой, если составляющие ее группы $M_{\lambda}$ свободные конечно порожденные, а отвечающие парам индексов $\lambda<\mu$ отображения $i_{\mu}^{\lambda}: M_{\lambda} \rightarrow M_{\mu}-$ вложения на прямые слагаемые. Здесь $\lambda$ - элементы некоторого частично упорядоченного множества, направленного в сторону возрастания.

В соответствии с [3] в случае, если базисные элементы в $M_{\lambda}$ могут быть выбраны одновременно таким образом, что для любой пары индексов $\lambda<\mu$ базис в $M_{\mu}$ распадается на подмножества $I(a)$, отвечающие элементам $a$ базиса в $M_{\lambda}$ и такие, что элементы $i_{\mu}^{\lambda}(a)$ совпадают с суммами элементов из $I(a)$, предельная группа $\lim _{\lambda}\left\{M_{\lambda}\right\}$ оказывается свободной. Этот результат был использован в [3], в частности, для того, чтобы установить свободность нульмерной групш когомологий с компактными носителями с коэффициентами в кольце главных идеалов любого локально компактногопространства и, как следствие, равенство нулю формально фигурировавшей в теории гомологий Бореля-Мура групш гомологий степени -1. Результат имеет первостепенное значение

Работа выполнена при финансовой поддержке Российского фонда фундаментальных исследований, грант № 03-01-00705. 
в конструкции свободных коцепей типа Чеха с компактными носителями в категории локально компактных пространств (см. [4, §3], [5, примечание к $\S 1]$, замечания после теоремы 3.1 в [6]).

Разумеется, класс локально свободных групп существенно шире класса свободных групп. В силу теоремы 19.2 в [7] к нему относятся, например, все прямые произведения бесконечных циклических групп (и подгрупшы таких произведений). В работе строятся локально свободные грушшы $\Sigma$, для которых $\operatorname{Hom}(\Sigma, Z)=0$. Такие групшы не содержат прямых слагаемых, изоморфных прямым произведениям бесконечных циклических групп (и не являются сепарабельными в смысле [2, § 87]).

Определенньй интерес представляют проективные системы груп, дуальные к простым индуктивным системам (см. конец работы).

Следующее определение и связанные с ним простые наблюдения содержатся в [7, $\S 16]$.

Абелева группа $M$ называется существенным расширением своей подгрупшы $E$, если $E \cap B \neq 0$ для всякой другой подгрупшы $B \subset M$. Очевидно достаточно, чтобы это свойство вьполнялось для всех циклических подгрупп $B \subset M$. Нетрудно убедиться в том, что ранг существенного расширения $M$ групшы $E$ тот же, что и у $E$. Группа $M$ без кручения тогда и только тогда является сушественным расширением подгрупшы $E$, когда факторгруппа $M / E$ - группа кручения. В ситуации, когда $E$ - подгруппа некоторой абелевой группы $\Sigma$, в $\Sigma$ имеется единственная подгруппа $M(E)$, служащая максимальным сушественным расширением $E$ в $\Sigma$.

Лемма 1. В әруппе без кручения $\Sigma$ подәруппь типа $M($ Е) сервантны.

В самом деле, из сказанного выше следует, что в группе $\Sigma$ без кручения $M(E)$ - максимальная содержащая $E$ подгруппа, для которой $M(E) / E$ - группа кручения. Если $n x \in M(E)$ для некоторого натурального числа $n$ и какого-то элемента $x \in \Sigma$, то порожденная $n x$ циклическая подгруппа имеет ненулевое пересечение с $E$ и потому для некоторого (кратного $n$ ) числа $m$ элемент $m x$ попадает в $E$. В силу указанного вьше свойства максимальности $M(E)$ элемент $x$ должен содержаться в этой группе.

Группу без кручения $\Sigma$ будем назьвать локально свободной, если ееподгрупшы $M(E)$ свободны для всех конечно порожденных подгрупп $E \subset \Sigma$.

Для элемента $x$ групшы без кручения $\Sigma$ такого, что $n x \in E$ при некотором $n$, пусть $h(x)$ - минимальное число среди всех таких $n$. Верхнюю грань в множестве всех таких чисел $h(x)$ будем обозначать через $h(E)$ (не исключено, вообще говоря, что $h(E)=\infty$ ) и называть высотой подгруппы $E$ в группе $\Sigma$.

ТЕОРемА 1. Для абелевой әруппы $\Sigma$ без кручения әквивалентны утверждения:

1) $\Sigma$ - локально свободная группа;

2) подгруппы конечного ранга в $\Sigma$ свободны;

3) счетные подгруппь группы $\Sigma$ свободны;

4) группы $M(E) / E$ конечны для всех конечно порохсденных подарупп $E \subset \Sigma$;

5) $h(E)<\infty$ для всех конечно порожсденных подарупп $E \subset \Sigma$;

6) $\Sigma$ - предел простой индуктивной системы әрупп. 
ДокАЗАТЕЛЬСТво. Факторгруппа группы $M$ конечного ранга по конечно порожденной подгруппе $E$ такого же ранга - группа кручения, поэтому $M$ - некоторое существенное расширение $E$, содержащееся в $M(E)$. Это обеспечивает эквивалентность первых двух утверж дений. Эквивалентность утверждений 2) и 3) - следствие того, что, с одной стороны, группы конечного ранга счетны, и, с другой стороны, всякая счетная группа, все подгруппы конечного ранга в которой свободны, сама свободна (теорема Понтрягина [7, теорема 19.1]). В [7, $§ 19]$ группы, для которых справедливо утверждение 3) теоремы, названы $\aleph_{1}$-свободными.

Для конечно порожденной подгруппы $E$ групшы без кручения $\Sigma$ группа $M(E)$ тогда и только тогда конечно порождена, когда группа кручения $M(E) / E$ конечна. Это означает эквивалентность утверждений 1) и 4). Из 4) следует 5): конечность $M(E) / E$ влечет, очевидно, конечность высоты $h(E)$. Из 5$)$ следует 1$)$. Групп $M(E)$, если она не свободна, есть объединение счетной последовательности содержащих $E$ и вложенных друг в друга свободных подгрупा $M_{1} \subset M_{2} \subset \cdots$ одного и того же конечного ранга, равного рангу $E$. Вследствие этого порядки элементов факторгрупп $M_{i} / E \subset M(E) / E$ с ростом $i$ возрастают, что означает неограниченность определяющих $h(E)$ чисел $h(x)$, т.е. бесконечность высоты $h(E)$ подгруппы $E$ в $\Sigma$.

Из 1) следует 6). В самом деле, включение $E_{1} \subset E_{2}$ влечет, очевидно, $M\left(E_{1}\right) \subset$ $M\left(E_{2}\right)$. Для конечно порожденных подгрупп $E_{1}, E_{2} \subset \Sigma$ имеем $M\left(E_{i}\right) \subset M\left(E_{1}+E_{2}\right)$, $i=1,2$. Следовательно, система подгруп $\{M(E)\}$, отвечающая всем конечно порожденньм подгрупам $E \subset \Sigma$, направлена по включению и

$$
\Sigma=\bigcup_{E \subset \Sigma} M(E)=\underset{E \subset \Sigma}{\underset{E \subset}{\lim }}\{M(E)\} .
$$

В силу леммы 1 подгрупшы $M(E)$ сервантны в $\Sigma$, а потому и в $M\left(E^{\prime}\right)$ для включений $E \subset E^{\prime}$. Так как групшы $M(E)$ конечно порождены, включения $M(E) \subset M\left(E^{\prime}\right)$ расщепляются [7, следствие 28.3], т.е. индуктивная система $\{M(E)\}$ является простой.

Наоборот, пусть

$$
\Sigma=\underset{\lambda}{\lim }\left\{M_{\lambda}\right\}
$$

- предел простой индуктивной системы. В этом случае можно считать, что $\Sigma$ - объединение своих подгрупп $M_{\lambda}$. Для любой конечно порожденной подгрупшы $E$ в $\Sigma$ найдется индекс $\lambda$, для которого $E \subset M_{\lambda}$. Для элемента $x \in \Sigma$ включение $n x \in E$ возможно, очевидно, лишш для $x \in M_{\lambda}$. Следовательно, $M(E) \subset M_{\lambda}$, и потому групша $M(E)$ свободна. Этим установлено, что утверждение 6 ) влечет 1). Теорема доказана.

ТЕОРема 2. Существуют локально свободные группы $\Sigma$, для которых $\operatorname{Hom}(\Sigma$, $Z)=0$.

Заметим, что в соответствии с утверждением 3 ) теоремы 1 всякая такая групп $\Sigma$ не содержит счетных прямых слагаемых. В частности, для любых конечно порожденных (а также для любых счетных) подгрупп $E \subset \Sigma$ сервантные в соответствии с леммой 1 подгрупшы $M(E) \subset \Sigma$ не являются прямыми слагаемыми. 
ДокАЗАТЕЛЬСТво теоремы начнем с описания некоторых вспомогательных конструкций, позволяющих на их основе строить нужные примеры.

Пусть $\mathscr{J}$ - некоторое несчетное множество мощности $p=p(\mathscr{J})$ и $\mathscr{L}=\{\lambda\}$ - частично упорядоченное по включению множество, состоящее из всех конечных подмножеств $\lambda \subset \mathscr{J}$ и имеющее, следовательно, ту же мощность $p$. В соответствии с [8] существует проективная система $\left\{N_{\lambda}, \Pi_{\mu}^{\lambda}\right\}$ бесконечных множеств $N_{\lambda}$, имеющих мощности $p\left(N_{\lambda}\right) \leqslant p$, с сюръективными проекциями $\Pi_{\mu}^{\lambda}: N_{\lambda} \rightarrow N_{\mu}$ для всех пар $\mu<\lambda$ и с пустым обратньм пределом $\lim _{\lambda}\left\{N_{\lambda}\right\}=\varnothing$. Аналогичная проективная система, состоящая из счетных множеств $N_{\lambda}$, определена в [9] над упорядоченным множеством $\mathscr{L}$ всех счетных трансфинитных чисел. Ниже предполагается, что $\left\{N_{\lambda}, \Pi_{\mu}^{\lambda}\right\}$ - одна из подобных проективных систем.

Пусть $Z\left(N_{\lambda}\right), \lambda \in \mathscr{L},-$ свободные группы с образуюшими элементами $N_{\lambda}$. Эти группы образуют проективную систему $\left\{Z\left(N_{\lambda}\right)\right\}$ с эпиморфными (и потому расщепляющимися) проекциями $\Pi_{\mu}^{\lambda}: Z\left(N_{\lambda}\right) \rightarrow Z\left(N_{\mu}\right)$ для всех $\mu<\lambda$.

Лемма 2. Имеет место соотношение

$$
\lim _{\grave{\lambda}}\left\{Z\left(N_{\lambda}\right)\right\}=0
$$

ДоКАЗАТЕЛЬСТво. Предположим, что предел отличен от нуля. Пусть $z-$ некоторьй отличный от нуля элемент этого предела, $z_{\lambda}$ - образ $z$ в группе $Z\left(N_{\lambda}\right)$ и $\left|z_{\lambda}\right|$ - носитель $z_{\lambda}$ в множестве $N_{\lambda}$, т.е. конечное множество элементов в $N_{\lambda}$, входящих в разложение $z_{\lambda}$ с ненулевыми коэффициентами. Имеем $\left|z_{\mu}\right| \subset \Pi_{\mu}^{\lambda}\left(\left|z_{\lambda}\right|\right)$ при $\mu<\lambda$. Фиксируем некоторое $\mu \in \mathscr{L}$, для которого $z_{\mu} \neq 0$ и, следовательно, $\left|z_{\mu}\right| \neq \varnothing$. Пусть $\mathscr{L}^{\prime}-$ конфинальная часть $\mathscr{L}$, отвечающая всем $\lambda \geqslant \mu$, и пусть $s_{\lambda}$ - прообразы в $\left|z_{\lambda}\right|$ множества $\left|z_{\mu}\right|$ при отображениях $\Pi_{\mu}^{\lambda}$. Множества $s_{\lambda}, \lambda \in \mathscr{L}^{\prime}$, составляют проективную систему $\left\{s_{\lambda}\right\}$, состоящую из компактных (конечных) множеств, поэтому $\lim _{\lambda}\left\{s_{\lambda}\right\} \neq \varnothing[10$, теорема 3.6 гл. VIII]. Поскольку $\mathscr{L}^{\prime}-$ конфинальная часть $\mathscr{L}$ и $s_{\lambda} \subset N_{\lambda}$ при $\lambda \geqslant \mu$, это противоречит тому, что $\lim _{\downarrow}\left\{N_{\lambda}\right\}=\varnothing$. Лемма доказана.

Для свободной группы $Z(N)$ с множеством образующих $N$ пусть $P(N)=Z(N)$ * $\operatorname{Hom}(Z(N), Z)$ - дуальная групша. Как правило, групшы $Z(N)$ и $P(N)$ взаимно дуальны, т.е. $Z(N)=P(N)$ *. Более точно [2, следствие 94.6], пока не известно примеров, когда это не так, причем, если взаимная дуальность групп $Z(N)$ и $P(N)$ имеет место для множеств $N$ некоторой мощности $p$, то она имеет место для всех множеств любой меньшей мощности $[2, \S 94]$. В конструкциях ниже условимся использовать проективные системы $\left\{N_{\lambda}, \Pi_{\mu}^{\lambda}\right\}$, для множеств $N_{\lambda}$, в которых имеет место указанная взаимная дуальность.

Вместе с включениями $\rho_{\lambda}^{\mu}=\left(\Pi_{\mu}^{\lambda}\right)^{*}: P\left(N_{\mu}\right) \rightarrow P\left(N_{\lambda}\right)$ групшы $P\left(N_{\lambda}\right)=Z\left(N_{\lambda}\right)^{*}$ образуют индуктивную систему (над тем же частично упорядоченным множеством индексов $\mathscr{L})$. Определим групу $\Sigma$ как предел этой системы:

$$
\Sigma=\lim _{\lambda}\left\{P\left(N_{\lambda}\right)\right\}
$$


Применяя к случаю $X_{\lambda}=P\left(N_{\lambda}\right)$ и $Y=Z$ известное соотношение

$$
\operatorname{Hom}\left(\lim _{\lambda}\left\{X_{\lambda}\right\}, Y\right)=\underset{\leftarrow}{\lim }\left\{\operatorname{Hom}\left(X_{\lambda}, Y\right)\right\}
$$

убеждаемся, что $\operatorname{Hom}(\Sigma, Z)=0$. Остается убедиться, что $\Sigma$ - локально свободная группа.

Как замечено во введении, в соответствии с теоремой 19.2 в [7] грушшы $P\left(N_{\lambda}\right)$ локально свободны. Кроме того, для всех пар индексов $\mu<\lambda$ отображения $\rho_{\lambda}^{\mu}$ индуктивной системы этих груп - расщепляющиеся включения. С учетом этого аргументы, примененные в заключительной части доказательства теоремы 1 , подтверждают, что $\Sigma-$ локально свободная группа. Теорема 2 доказана.

Заметим, что $\operatorname{Hom}(X, Z)=0$ для грушшы $X=\Pi_{1}^{\infty} Z / \bigoplus_{1}^{\infty} Z$ (это следует из взаимной дуальности $\Pi_{1}^{\infty} Z$ и $\bigoplus_{1}^{\infty} Z$ при дуализации точной последовательности $0 \rightarrow$ $\left.\bigoplus_{1}^{\infty} Z \rightarrow \Pi_{1}^{\infty} Z \rightarrow X \rightarrow 0\right)$. Однако группа $X$ не локально свободна: образ в $X$ элемента $\left\{x_{n}\right\} \in \Pi_{1}^{\infty} Z$, для которого $x_{n}=n$ !, имеет в $X$ бесконечную высоту.

В заключение отметим очевидную связь аппроксимации локально свободньг групп простьми индуктивньми системами с задачей описания обратных пределов свободных конечно порожденных групш.

Как известно, мощность обратного предела $A=\lim _{i}\left\{A_{i}\right\}$ нетривиальной башни групп с эпиморфньми проекциями $A_{i+1} \rightarrow A_{i}$ всегда не менее континуума. Это весьма простое наблюдение оказывается существенным при выяснении условий, обеспечиваюших изоморфизм гомологий Чеха, обладаюших свойством точности, гомологиям Стинрода-Ситникова (см., например, теорему 4 в [4]), как и при выявлении связанньх с приложениями свойств функтора $\lim ^{1}$ (см. [11], [12, §1], [6, §1]).

Совсем иная картина наблюдается в классе несчетных проективных систем. Поскольку группа $\Pi_{1}^{\infty} Z$ локально свободна и дуальна с $\bigoplus_{1}^{\infty} Z$, проективная система, дуальная к аппроксимирующей групу $\Pi_{1}^{\infty} Z$ простой индуктивной системе, составлена конечно порожденньми свободными группами с эпиморфными проекциями друг в друга, но имеет своим пределом (в силу соотношения (*) выше) счетную группу $\bigoplus_{1}^{\infty} Z[6$, пример 1.2]. Отвечая на вопрос, поставленньй в [13], авторы [9] предъявляют пример проективной системы (над счетными трансфинитными числами) счетномерных векторных пространств с эпиморфными проекциями, имеющей нулевой предел. Ясно, что векторные пространства в этом примере могут быть заменены на счетнопорожденные свободные группы. Использующая результат [8] конструкция, примененная в лемме 2 вьше, является усовершенствованием примера 1.1 в [6].

При независимом от [8] и [9] подходе Т. Л. Куренковой [14] изучались различные свойства обратных пределов проективных систем однородных вполне разложимых групш конечного ранга с расщепляющимися эпиморфными проекциями. Ею впервые был сконструирован пример такой системы с нулевьм пределом, составленной свободными группами конечного ранга. В соответствии с соотношением (*) такими примерами оказьваются проективные системы, дуальные к простым индуктивньм системам, представляющим любую локально свободную группу $\Sigma$, для которой $\operatorname{Hom}(\Sigma, Z)=0$. 


\section{СПИСОК ЦИТИРОВАННОЙ ЛИТЕРАТУРЫ}

[1] Курош А. Г. Теория групп. М.: Наука, 1967.

[2] Фукс Л. Бесконечные абелевы группы. Т. 2. М.: Мир, 1977.

[3] Kaup L., Keane M.S. Induktive Limiten endlich erzeugter freier Moduln // Manuscr. Math. 1969. V. 1. № 1. P. 9-21.

[4] Скляренко Е.Г. Теория гомологий и аксиома точности // УМН. 1969. Т. 24. №5. C. $87-140$.

[5] Скляренко Е. Г. К теории обобщенных многообразий // Изв. АН СССР. Сер. матем. 1971. T. 35. № 4. C. 831-843.

[6] Скляренко Е. Г. Некоторые применения функтора $\lim ^{1} / /$ Матем. сб. 1984. Т. 123. №3. C. $369-390$.

[7] Фукс Л. Бесконечные абелевы группы. Т. 1. М.: Мир, 1974.

[8] Henkin L. A problem on inverse mapping systems // Proc. Amer. Math. Soc. 1950. V. 1. № 2. P. 224-225.

[9] Higman G., Stone A. H. On inverse systems with trivial limits // J. London Math. Soc. 1954. V. 29. P. 233-236.

[10] Стинрод Н., Эйленберг С. Основания алгебраической топологии. М.: ИЛ, 1958.

[11] Gray B. I. Space of the same $n$-type for all $n$ // Topology. 1966. V. 5. № 3. P. 241-243.

[12] Харлан А. Э. Локальные гомологии и когомологии, гомологическая размерность и обобщенные многообразия // Матем. сб. 1975. Т. 96. № 3. С. 347-373.

[13] Zelinsky Rings with ideal nuclei // Duke Math. J. 1951. V. 18. P. 431-442.

[14] Куренкова Т. Л. Счетные группы как обратные пределы групп конечного ранга // Избранные вопросы алгебры, геометрии и дискретной математики. М.: Изд-во Моск. ун-та, 1988. C. $47-57$. 